\title{
Anesthesia under cardiopulmonary bypass for video assisted thoracoscopic wedge resection in patient with spontaneous pneumothorax and contralateral post-tuberculosis destroyed lung
}

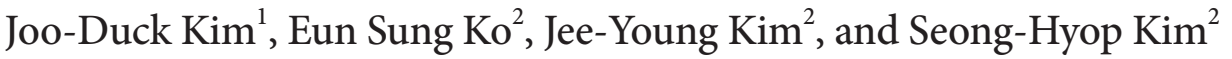 \\ Department of Anesthesiology and Pain Medicine, ${ }^{1}$ Kosin University Gospel Hospital, Busan, ${ }^{2}$ Konkuk University Medical Center, \\ Konkuk University School of Medicine, Seoul, Korea
}

Pneumothorax with contralateral destroyed lung is a rare condition. However, the anesthetic management poses significant risks and can be quite challenging because conventional one-lung ventilation (OLV) for wedge resection in pneumothorax with contralateral destroyed lung is impossible.

A 69-year-old man with height $167 \mathrm{~cm}$ and weight $57 \mathrm{~kg}$ was scheduled for video assisted thoracoscopic surgery (VATS) due to pneumothorax in left lung. A thoracostomy tube was inserted but failed to resolve completely the pneumothorax and persistent air leakage remained. The patient had a history of pulmonary tuberculosis, 16 years ago. A chest X-ray as preoperative evaluation showed pneumothorax with chest tube in the left lung and the right lung was destroyed with marked volume loss with deviation of trachea to right. Computed tomography showed multiple bullae, fibrotic bands and pneumothorax with chest tube in the left lung. Further, a destroyed lung with bronchopleural fistula (BPF) at the right lung was visible (Fig. 1).

Due to contralateral post-tuberculosis destroyed lung and BPF, OLV at dependent lung was impossible. VATS for pneumothorax under cardiopulmonary bypass (CPB) was decided after discussion between anesthesiologists and thoracic surgeons.

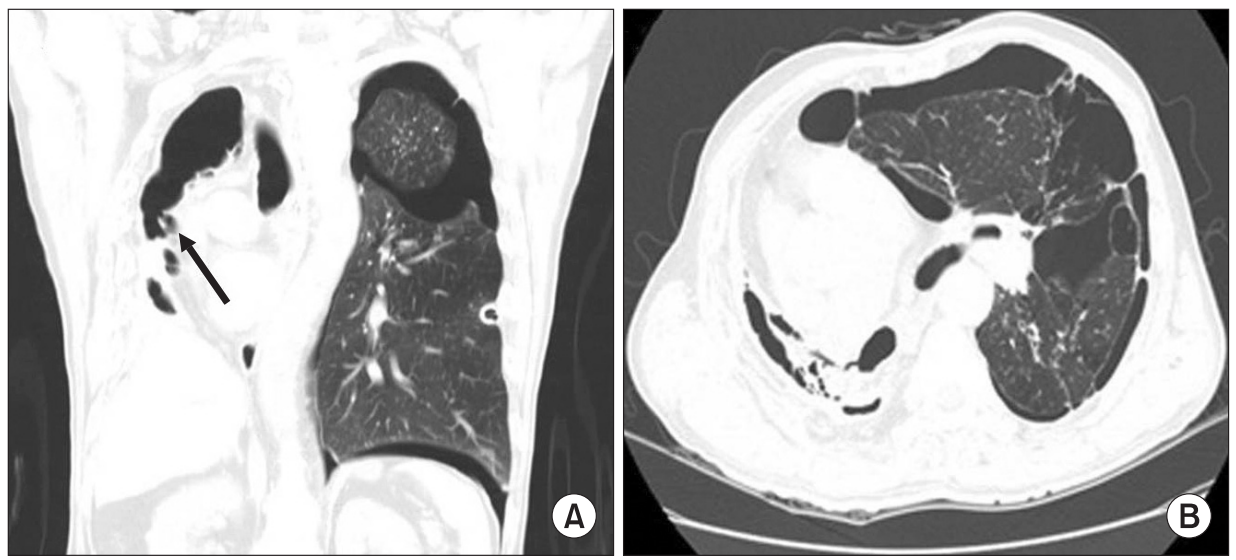

Fig. 1. (A) Coronal plane of the chest computed tomographic scan showed pneumothorax with chest tube in the left lung and a destroyed lung with bronchopleural fistula (arrow) at the right lung. (B) Axial plane of the chest computed tomographic scan showed fibrotic bands and multiple bullae with pneumothorax in the left lung and a destroyed lung at the right lung.

Corresponding author: Seong-Hyop Kim, M.D., Department of Anesthesiology and Pain Medicine, Konkuk University Hospital, 120-1, Neungdong-ro, Hwayang-dong, Gwangjin-gu, Seoul 143-729, Korea. Tel: 82-2-2030-5454, Fax: 82-2-2030-5449, E-mail: yshkim75@naver.com (c) This is an open-access article distributed under the terms of the Creative Commons Attribution Non-Commercial License (http:// creativecommons.org/licenses/by-nc/3.0/), which permits unrestricted non-commercial use, distribution, and reproduction in any medium, provided the original work is properly cited. 
Standard monitoring was started. The right radial artery was cannulated with $20 \mathrm{G}$ for continuous monitoring of systemic blood pressure. For the patient's safety, CPB was firstly established before anesthesia induction. Femoral artery cannula and femoral venous cannula were inserted through the left femoral artery and vein, respectively, by thoracic surgeons. Cannulation for $\mathrm{CPB}$ was established under sedation with spontaneous ventilation, using intermittent bolus injection of midazolam $1 \mathrm{mg}$ and target controlled infusion (TCI) of remifentanil (plasma concentration, $1 \mathrm{ng} / \mathrm{ml}$ ). During cannulation, the patient was supplied with oxygen $5 \mathrm{~L} / \mathrm{min}$ via face mask. Pulse oximetry was maintained above $95 \%$ and bispectral index (BIS) value showed 80 .

After establishment of femoro-femoral CPB, partial CPB was initiated with pump flow rate $1.4 \mathrm{~L} / \mathrm{min} / \mathrm{m}^{2}$. Under partial CPB, general anesthesia was induced with propofol $40 \mathrm{mg}$ and TCI of remifentanil $1 \mathrm{ng} / \mathrm{ml}$ (plasma concentration). During manual mask ventilation, oxygen $10 \mathrm{~L} / \mathrm{min}$ was supplied. There was no difficulty in manual ventilation though continuous air leakage in the chest bottle existed. The trachea was intubated after paralysis was achieved with $60 \mathrm{mg}$ of rocuronium. Immediately after intubation, a right main bronchus blockade was performed using Arndt endobronchial blocker, due to the presence of BPF in the right lung.

Anesthesia was maintained according to BIS value between 40 and 60 with $1.0 \%$ sevoflurane and remifentanil $5 \mathrm{ng} / \mathrm{ml}$ (plasma concentration) with TCI. The patient was mechanically ventilated with volume-controlled ventilation, tidal volume $350 \mathrm{ml}$ and respiratory rate 12 cycles/min with $100 \%$ oxygen. Arterial blood gas analysis showed $\mathrm{pH} 7.45$, partial pressure of arterial carbon dioxide $42.6 \mathrm{mmHg}$, partial pressure of arterial oxygen $489.5 \mathrm{mmHg}$ and arterial oxygen saturation $\left(\mathrm{SaO}_{2}\right)$ 99.9\% with hemodynamic stability. For adequate venous drainage during $\mathrm{CPB}$, superior vena cava (SVC) cannula was inserted through the right internal jugular vein. The central venous catheterization was performed at the left internal jugular vein. The patient was then turned into right lateral decubitus position for VATS procedure. Normothermic CPB was resumed with beating heart. The pump flow was set at 1.7 $\mathrm{L} / \mathrm{min} / \mathrm{m}^{2}$. Mechanical ventilation was stopped for satisfactory surgical field. The patient's hemodynamic was stable with mean systemic arterial pressure at $70-80 \mathrm{mmHg}$ and oxygenation was maintained above $98 \%$. During CPB, cerebral oximetry showed above 65. After completion of VATS for wedge resection, the patient was weaned from CPB uneventfully.

In the present case, there were two considerations for the patient's safety and success of VATS. One was how we could maintain adequate oxygenation throughout intra-operative period, since the nondependent left lung had pneumothorax with multiple bullae and the dependant right lung was destroyed with BPF after pulmonary tuberculosis. In addition, the other was how we could establish satisfactory surgical field during intra-operative period, because lung collapse was mandatory to facilitate intrathoracic surgical procedure. To meet two considerations, $\mathrm{CPB}$ was chosen in the present case.

Before the decision of using $\mathrm{CPB}$, different anesthetic managements, including selective ventilation of one lobe using endobronchial blocker, high-frequency jet ventilation (HFJV) and intermittent apnea were also considered. However, the patient in the present case could not be a candidate for single lobe ventilation due to patient's multiple bullae with pneumothorax in the left lobe. If we applied single lobe ventilation to the patient, the potential risk of barotrauma in emphysematous lung would increase. HFJV and intermittent apnea also has been used in lung surgery patients with limited contralateral lung function [1,2]. With regards to HFJV and intermittent apnea, they may not work well with severely emphysematous lung, like the present case. Also, visualization during VATS may be difficult due to air trapping, even with a non-ventilated lung.

The patient had BPF as complication of pulmonary tuberculosis at the destroyed right lung in the present case. To prevent wasted ventilation through the fistula during mechanical ventilation and contamination from the posttuberculosis destroyed right lung to the left lung, we performed isolation of the right lung using Arndt endobronchial blocker. In view of trachea's deviation to right, it was preferred, rather than double lumen tube in order to reduce the complication to the tracheaobronchial system.

When total CPB was needed, $21 \mathrm{Fr}$ femoral venous cannula could not offer sufficient venous drainage to $C P B$ venous reservoir. Therefore, SVC cannula was inserted through the right internal jugular vein with $20 \mathrm{Fr}$ cannula. For adequate venous drainage, the venous cannula positioning is very important and transesophageal echocardiography (TEE) is useful to guide and verify the correct positioning. In the present case, femoral venous cannula was firstly positioned in the right atrium for better venous drainage, until SVC cannulation, and then femoral venous cannula was repositioned into the inferior vena cava by TEE guidance. Fortunately, patient's $\mathrm{SaO}_{2}$ was maintained mostly above $99 \%$ with partial CPB flow of $1.7 \mathrm{~L} / \mathrm{min} / \mathrm{m}^{2}$ with no mechanical ventilation during wedge resection. Cerebral oximetry was monitored for evaluation of adequate oxygenation and venous drainage to brain. Cerebral oximetry during partial CPB also showed above 65 .

In conclusion, $\mathrm{CPB}$ is a feasible and beneficial technique to maintain adequate oxygenation while providing suitable surgical conditions in case of pneumothorax with contralateral post-tuberculosis destroyed lung. 


\section{References}

1. Terzi A, Furlan G, Gorla A, Falezza G, Attino M, Calabrò F. Lung resection on single residual lung after pneumonectomy for bronchogenic carcinoma. Thorac Cardiovasc Surg 1997; 45: 273-6.

2. Donington JS, Miller DL, Rowland CC, Deschamps C, Allen MS, Trastek VF, et al. Subsequent pulmonary resection for bronchogenic carcinoma after pneumonectomy. Ann Thorac Surg 2002; 74: 154-8. 